\title{
Notice of retraction
}

The Editors of Molecular \& Cellular Toxicology

(C) The Korean Society of Toxicogenomics and Toxicoporeteomics and Springer 2010

Erratum to: Molecular \& Cellular Toxicology (2010) 6(1):41-49.

DOI 10.1007/s13273-010-0006-5

The Editors of Molecular \& Cellular Toxicology and the publisher hereby retract the article entitled "Sodium nitroprusside induces autophagic cell death in glutathione-depleted osteoblasts" by Min Jeong Son et al. which appeared in Molecular \& Cellular Toxicology (2010) 6(1):41-49. Due to an error in our editorial database system, this article was not excluded from our publication list on the March issue.

The Editors of Molecular \& Cellular Toxicology, The Korean Society of Toxicogenomics and Toxicoproteomics 